\title{
Along came a spider: an unusual organism identified in a peritoneal dialysis patient, a case report and literature review
}

\author{
Victoria Jane Carnall ${ }^{1 *}$, Stephanie Murdock², Cressida Auckland ${ }^{2}$ and Christopher J. Mulgrew ${ }^{1}$
}

\begin{abstract}
Background: Peritoneal dialysis-associated peritonitis can uncommonly be caused by fungal infections. When they do present, they are associated with significant mortality and morbidity. We describe a case where a sample of peritoneal dialysate fluid grew Rhodotorula muciliginosa, a yeast organism present in the normal environment which has previously been reported as rarely causing peritonitis. We believe this is the first case where the Rhodotorula spp. and its origin has been identified.

Case presentation: A 20 year old male grew Rhodotorula muciliginosa from his peritoneal dialysis fluid on three separate occasions when a fluid sample was sent following a disconnection and subsequent set change. He was not systemically unwell and his peritoneal dialysate was clear. As Rhodotorula spp. is exceedingly difficult to treat our patient had his Tenchkoff catheter removed. Subsequent samples of soil and sand from his bearded dragon and Chilean tarantula cases, kept in his bedroom where dialysis occurred, were tested. The tarantula sand was identified as the source of the Rhodotorula spp. Of note, Candida was isolated from sand from the bearded dragon case. Once his Tenchkoff was removed he was treated with an intravenous course of antifungal therapy. He has since had a new Tenchkoff catheter inserted and recommenced PD following education around pets and hygiene.

Conclusions: In this era where people are keeping increasingly rare and unusual wildlife in their homes, this case highlights the need for clinician and nursing staff awareness of a patient's home environment and hobbies when they are undergoing peritoneal dialysis. Sand from our patient's tarantula case grew the colonising organism but interestingly soil from his bearded dragon case also isolated candida. This can also cause difficult to treat peritonitis.
\end{abstract}

Keywords: Peritonitis, Pets, Fungal infection, Rhodotorula, Zoonotic, Case report

\section{Background}

Fungal infections are an uncommon but serious cause of PD (Peritoneal Dialysis)-associated peritonitis [1]. The incidence worldwide is heterogenous with rates varying from 2 to $23.8 \%$ [2]. In the United Kingdom an audit study reported a fungal peritonitis rate of $0.0099 /$ patient year from centres not using prophylaxis and a lower rate of $0.0032 /$ patient year in centres using daily oral

\footnotetext{
* Correspondence: v.carnall@nhs.net

${ }^{1}$ Exeter Kidney Unit, Royal Devon and Exeter Foundation Trust, Exeter, Devon, UK

Full list of author information is available at the end of the article
}

fluconazole [3]. Despite its rarity fungal peritonitis is important due to its associated higher rates of catheter loss, morbidity and mortality [3]. Candida species are the commonest pathogen isolated in fungal peritonitis, accounting for $70-90 \%$ of the cases described [3]. Patients at particular risk for fungal peritonitis are those who have had previous bacterial peritonitis, been on prolonged antibiotic treatment, have gynaecological or bowel sources of infection, those who are immunosuppressed, have diabetes mellitus, are malnourished, or have a prolonged time on PD [2,3]. We describe a case where Rhodotorula muciliginosa was isolated from a 
patients' peritoneal dialysate fluid following an accidental set disconnection. This organism is a pigmented yeast, previously regarded as an environmental organism of little pathogenic significance, often present on the skin as well as in sputum, urine and faeces [4]. In the environment Rhodotorula spp. favours warm, wet locations, such as shower curtains and toothbrushes [5]. Increasing evidence suggests its emergence as an opportunistic pathogen, particularly in the immunocompromised population. Published reports indicate Rhodotorula spp. as the likely causative agent in catheter-associated fungaemia and peritonitis, most relevant to our renal patients $[6,7]$, but also other infections such as meningitis, endocarditis, and prosthetic joint infections [4]. Rhodotorula spp. causing PD peritonitis has been reported in eight papers, none of which have identified the source of the organism. One paper in 1983 identified a point source outbreak of three cases in Canada attributable to Rhodotorula muciliginosa isolated from environmental sampling, all three patients had their catheters removed [8]. A paediatric patient on APD (Automated Peritoneal Dialysis) had Rhodotorula muciliginosa isolated from their dialysate fluid, they were treated with IV antifungal therapy and tube removal [9]. Five adult cases have been reported; one patient punctured his PD bag and continued to use it, he was successfully treated with antifungals. Three further patients had antifungal treatment and subsequent catheter removal $[7,10-12]$. The final patient had antifungal treatment and catheter exchange [13]. Unal et al.analysed the clinical presentations, aetiology and treatment of fungal peritonitis in their hospital. Only one case of Rhodotorula muciliginosa was identified in their case report of 21 cases. Their patient was treated with amphotericin and the catheter removed [14]. Most reported cases of Rhodotorula spp. infections are due to muciliginosa, with an incidence of $68.6 \%$ reported in a systematic review by Ioannou et in 2018, with R.glutinis and R.minuta being less common [15]. Our case is the first which has grown Rhodotorula spp. from peritoneal dialysate fluid as well as identifying the likely source.

\section{Case presentation}

A 20 year old man with chronic kidney disease stage 5 secondary to chronic interstitial nephritis had been performing continuous ambulatory peritoneal dialysis for six months before having an inadvertent dialysis catheter disconnection.

As per unit protocol he underwent a set change with concurrent prophylactic IP vancomycin administration. A sample of peritoneal dialysis fluid was sent for microscopy and culture. Initial microscopy demonstrated a white cell count of only $10 \times 10^{6} / \mathrm{l}$, this count was not raised therefore a differentiated cell count was not performed. No organisms were seen on Gram stain. He was clinically well with no abdominal pain, fevers or cloudy bags. The cultured peritoneal dialysis fluid grew Rhodotorula muciliginosa (Fig. 1). A further two peritoneal fluid samples sent up to 7 days after the first sample also grew the same Rhodotorula spp. suggesting colonisation. Sensitivity tests were performed at the Mycology Reference Laboratory in Bristol using microtitre broth dilution according to CLSI M-27-A3 protocol. This test showed our isolate had MIC levels of $0.25 \mathrm{mg} / \mathrm{L}$ for amphotericin, $0.50 \mathrm{mg} / \mathrm{L}$ for posoconazole and $4 \mathrm{mg} / \mathrm{L}$ for voriconazole. Our isolate was considered sensitive to amphotericin only. The patient's C-reactive protein peaked at $30 \mathrm{mg} / \mathrm{l}$ (normal range $0-5 \mathrm{mg} / \mathrm{l}$ ) with a mildly elevated white cell count of $13.8 \times 10^{9} / \mathrm{L}$ comprised of $11.8 \times 10^{9} / \mathrm{L}$ neutrophils and $0.8 \times 10^{9} / \mathrm{L}$ lymphocytes. On microbiology advice, given the potential for the patient to develop fungal peritonitis, he was admitted for removal of his peritoneal dialysis catheter and commencement of IV amphotericin.

An investigation into the potential source of the Rhodotorula spp. colonisation was commenced. It was noted that he kept a Chilean rose tarantula (Fig. 2), an Indian ornamental tarantula, and a bearded dragon, in the room where he performed his dialysis.

Samples were sent from the sand and soil environments within the animal habitats. Microbiological culture of the sand from the tarantula case grew Rhodotorula muciliginosa, identifying the likely source. Interestingly, Candida spp. was also isolated from sand from the bearded dragon case, which can also cause devastating peritonitis.

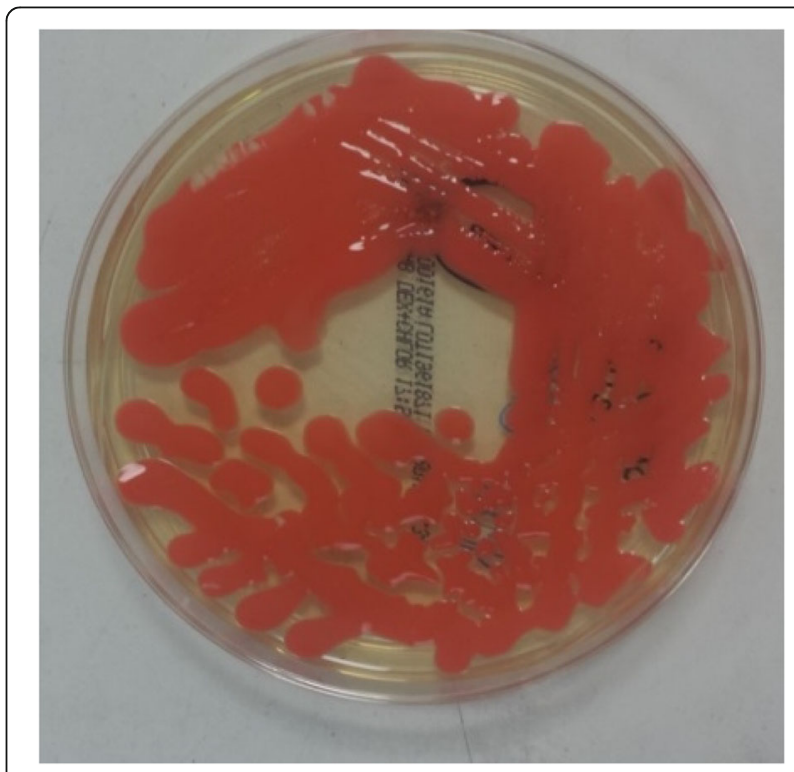

Fig. 1 Shiny salmon-pink Rhodotorula colonies on Sabouraud medium 


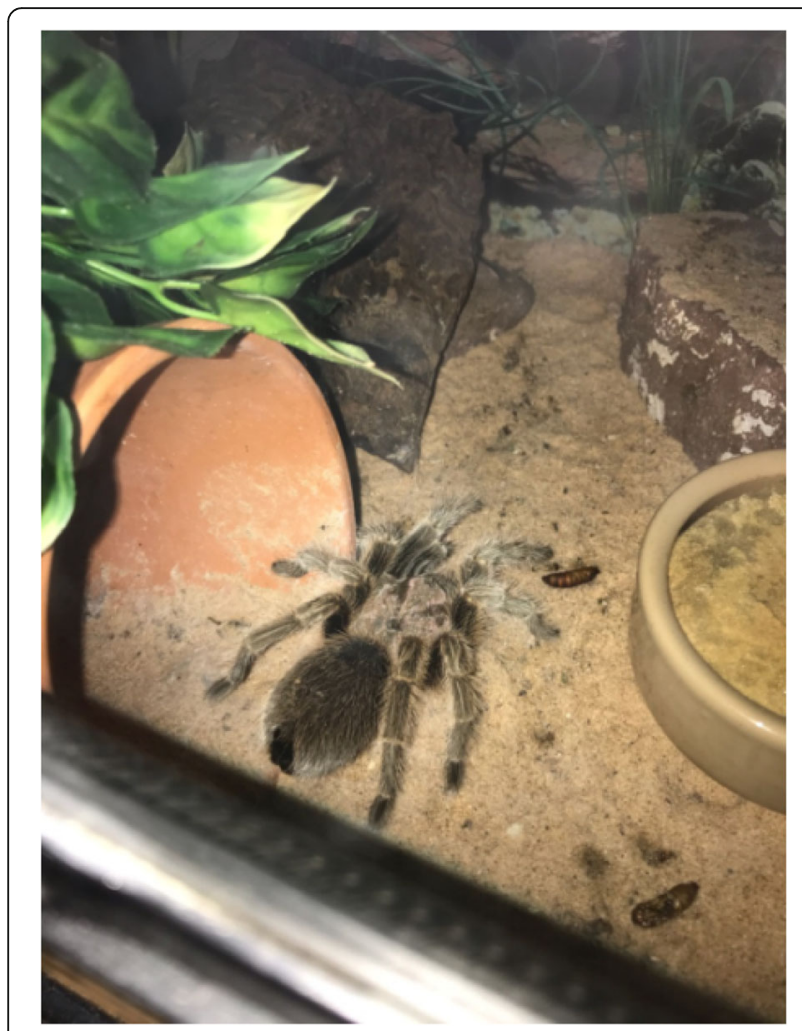

Fig. 2 Chilean Rose Tarantula kept in the room where peritoneal dialysis exchanges occurred

Given our patients preserved urine output, he was able to remain off renal replacement therapy for two months following completion of the Amphotericin course. The patient was pleased that dialysis could be held, and peritoneal dialysis recommenced now a source had been identified, as he wished to avoid haemodialysis. A further Tenchkoff catheter was inserted and dialysis resumed. In the interim, the cases housing the tarantulas and bearded dragon were moved to another area of the house, and a thorough deep clean of his room completed.

\section{Discussion and conclusions}

We describe the first case where Rhodotorula muciliginosa has been isolated in peritoneal dialysate fluid and the likely source, the cage of a Chilean rose tarantula, identified. Zoonotic infections in humans originating from tarantulas are rare. If they occur it is usually a result of tarantula setae (hairs) penetrating the skin or eyes, or following a bite. Mechanical irritation or hypersensitivity reactions are more likely to occur rather than true infection [14]. A literature search revealed just one case of Aspergillus infection following ocular injury from setae in a dog [15]. Studies on the carriage of saprophytic fungi by arthropods, including spiders, has demonstrated a $40 \%$ carriage rate for Aspergillus spp. however Rhodotorula spp. was not isolated [16].

Fungal infections are becoming an increasing healthcare burden. Unfortunately the number of causative pathogens, particularly affecting immunocompromised patients are growing, without an expansion in treatment options. This point was highlighted by Giacobino et al. who presented their fungal peritonitis data from a Brazilian hospital. They demonstrated causative organisms arising from a diverse group of yeasts and more importantly a variation in susceptibility to antifungals, and a significant resistance to fluconazole, causing increased difficulty in treatment [17]. The overall rates of reported Rhodotorula spp. infections are increasing, this is likely due to an increasing number of immunocompromised patients as well as advances in medicine resulting in more transplantation, more extensive $\mathrm{CVC}$ use and increasing use of broad spectrum antibiotics [4]. Rhodotorula spp. fungaemia has been associated with a crude mortality of $20 \%$ [18]. This figure is of particular concern with Rhodotorula spp. demonstrating resistance to first and second line generation triazoles, only intermediate sensitivity to amphotericin and only full sensitivity to flucytosine. As Rhodotorula spp. is rare the clinical breakpoint for each anti-fungal has not been published [19].

Amphotericin B was used in all the reported cases of Rhodotorula spp. peritonitis, our report being the only case where the source was identified. Amphotericin B demonstrates concentration-dependant fungicidal activity versus candida, with a ratio of the maximum serum concentration to MIC $\left(\mathrm{C}_{\max } / \mathrm{MIC}\right)$ of 2.4 or higher giving optimal efficacy [20]. The maximum concentration of liposomal amphotericin in peritoneal fluid to $\mathrm{MIC}\left(\mathrm{PF}_{\max } /\right.$ MIC) is probably a better correlate of efficacy when treating PD infections. Amphotericin B is $90 \%$ protein-bound and diffuses poorly into the peritoneal fluid, with correspondingly low $\mathrm{PF}_{\max }$ levels. The more commonly used liposomal formulation has even more limited peritoneal fluid penetration and peritoneal levels may indeed be subtherapeutic, as summarised in Table 1 . In our case, the MIC of Rhodotorula muciliginosa was $0.25 \mathrm{mg} / \mathrm{L}$, giving a ratio of $\mathrm{PF}_{\max } / \mathrm{MIC}$ of between 0.5 and 3, where the optimal level is 2.4. The efficacy of liposomal amphotericin might therefore be in doubt, although our patient responded to liposomal amphotericin along with catheter removal. Intra-peritoneal administration of amphotericin is no longer recommended due to peritoneal irritation and fibrosis [7].

The significance of Rhodotorula spp. as a pathogenic organism in the renal population is not isolated to peritoneal dialysis as the organism has also been isolated from central venous catheters [25], suggesting the haemodialysis population could also be at risk. 
Table 1 Peritoneal concentrations of Amphotericin B [20-24]

\begin{tabular}{llllll}
\hline Author and Year & $\begin{array}{l}\text { Number in } \\
\text { study }\end{array}$ & Antifungal Agent & $\begin{array}{l}\text { Serum concentration } \\
\text { (mg/L) }\end{array}$ & $\begin{array}{l}\text { Peritoneal concentration } \\
\text { (mg/L) }\end{array}$ & $\begin{array}{l}\text { Peritoneal/serum } \\
\text { penetration ratio }\end{array}$ \\
\hline Polak 1979 [21] & NR & Amphotericin B & $0.4-1.7$ & $0.22-0.32$ & $19-55 \%$ \\
Petersen 1978 [22] & 1 & Amphotericin B & 0.52 (predose) & 0.44 (predose) & $85 \%$ \\
& & & 1.5 (postdose) & 0.78 (postdose) & $52 \%$ \\
Van der Voort & 21 & Amphotericin B & 0.25 (median) & 0.12 (median) & $41 \%$ \\
2007 [23] & & & & & $N$ \\
Weiler 2008 [20] & 1 & Liposomal amphotericin & NR & 0.258 & $N R$ \\
\hline
\end{tabular}

NR Not reported

The current ISPD guidelines suggest that in cases of fungal peritonitis prompt catheter removal probably improves outcome and reduces mortality with anti-fungal agents continued after catheter removal for at least 2 weeks [26]. Research suggests permanent transfer to haemodialysis is frequent following an episode of fungal peritonitis although a return to peritoneal dialysis is possible, particularly in less fragile patients. [2]. Although our patient did not meet the criteria for peritonitis the severe morbidity and mortality associated with fungal peritonitis lead us to treat the patients' colonisation in the same manner. The ISPD guidelines also suggest a home visit by a PD nurse to detect problems with exchange technique, adherence to protocols, and to identify other environmental and behaviour issues that increase the risk of infections such as pets be carried out [26].

With unusual animal species becoming ever more popular as household pets the awareness of these as a source of infection is key. In our case, theoretically the bearded dragons case, which isolated Candida, was also a potential source. A good understanding of the patient's background, home environment, and the potential for animals to be a source for colonisation and peritonitis is vital to reduce the risk of morbidity and mortality, particularly in dialysis patients.

\section{Acknowledgements}

Kevin Wilson (Homecare nurse specialist) for the photography and sample collection.

\section{Authors' contributions}

SM wrote the microbiology sections of the manuscript. VC wrote the nephrology sections of the manuscript and collated the two sections. CM and CA critically reviewed and analysed the nephrology and microbiology components of this manuscript respectively. All authors read and approved the final manuscript.

\section{Funding}

Not applicable.

\section{Availability of data and materials}

Not applicable.

Ethics approval and consent to participate Not applicable.
Consent for publication

Written consent was obtained from the patient for publication of this Case Report and accompanying images.

\section{Competing interests}

The authors declare they have no competing interests.

\section{Author details}

${ }^{1}$ Exeter Kidney Unit, Royal Devon and Exeter Foundation Trust, Exeter, Devon, UK. ${ }^{2}$ Microbiology Department, Royal Devon and Exeter Foundation Trust, Exeter, Devon, UK

Received: 6 May 2020 Accepted: 12 October 2020

Published online: 11 November 2020

\section{References}

1. Matuszkiewicz-Rowinska J. Update on fungal peritonitis and its treatment. Perit Dial Int. 2009;29(Suppl 2):161-5.

2. Auricchio $S$, et al. Fungal peritonitis in peritoneal dialysis: a 34-year single centre evaluation. Clin Kidney J. 2018;11(6):874-80.

3. Hu S, et al. Fungal peritonitis in peritoneal dialysis: 5-year review from a North China center. Infection. 2019:47:1204-7.

4. Falces-Romero I. Isolation of Rhodotorula muciliginosa from blood cultures in a tertiary care hospital. Mycoses. 2017;61:35-9.

5. Miles R. Predictors and outcomes of fungal peritonitis in peritoneal dialysis patients. Kidney Int. 2009;76:622-8.

6. Tuon FF, Costa SF. Rhodotorula infection a systematic review of 128 cases from literature. Revistalberoamericana De Micologia. 2008;25:135-40.

7. Unal A, et al. CAPD-related peritonitis caused by Rhodotorula muciliginosa. Perit Dial Int. 2009;29(5):581-2.

8. Eisenberg ES, et al. Rhodotorularubra peritonitis in patients undergoing continuous ambulatory peritoneal dialysis. Am J Med. 1983;75:349-52.

9. Soylu A, et al. Unusual cause of peritonitis during peritoneal dialysis Rhodotorularubra and amphotericin B. PediatrNephrol. 2004;19(12):1426-8.

10. de Zoysa JR, et al. Successful treatment of CAPD peritonitis caused by Rhodotorula muciliginosa. Perit Dial Int. 2001;21(6):627-8.

11. Verdugo FJ, et al. Fungal peritonitis due to Rhodotorula muciliginosa in a patient with automated peritoneal dialysis: literature review. RevistaChilena De Infectologia. 2016;33(2):222-5.

12. Pennington JC III, Hauer K, Miller W. RhodotorulaRubra peritonitis in an HIV+ patient on CAPD. Del Med J. 1995;67(3):184

13. Franconieri F, et al. Fungal peritonitis caused by Rhodotorula muciliginosa in a CAPD patient treated with liposomal amphotericin B a case report and literature review. Perit Dial Int. 2018:38(1):69-73.

14. Unal A, et al. Fungal peritonitis in peritoneal dialysis: an analysis of 21 cases. IntUrolNephrol. 2011;43(1):211-3.

15. Ioannou P, et al. Rhodotorula species infections in humans: a systematic review. Mycoses. 2018;62:90-100

16. Reed $Z$, et al. Tarantula hair keratoconjunctivitis with concurrent fungal infection in a rat terrier. J Am AnimHospAssoc. 2016;52(6):392-7.

17. Giacobino J, et al. Fungal peritonitis in patients undergoing peritoneal dialysis (PD) in Brazil: moleculat identification, biofilm production and antifungal susceptibility of the agents. Med Mycol. 2016:54:725-32.

18. Greif MD, Currah RS. Patterns in the occurrence of saprophytic fungi carried by arthropods caught in traps baited with rotted wood and dung. Mycologia. 2007;99(1):7-19. 
19. "Antifungal Agents: Breakpoint Tables for the Interpretation of MICs." European Committee on Antimicrobial Susceptibility Testing, 1 Mar. 2017, Accessed on $28^{\text {th }}$ September 2019 at: www.eucast.org/fileadmin/src/media/ PDFs/EUCAST_files/AFST/Clinical_breakpoints/Antifungal_breakpoints_v_ 8.1_March_2017.pdf.

20. Weiler $\mathrm{S}$, et al. Levels of amphotericin B lipid formulations in ascites. J AntimicrobChemother. 2008;62(5):1163-4.

21. Polak A. Pharmacokinetics of amphotericin B and flucytosine. Postgrad Med J. 1979:55(647):667-70

22. Peterson $L R$, et al. Therapy of candida peritonitis: penetration of amphotericin B into peritoneal fluid. Postgrad Med J. 1978:54(631):340-2.

23. Van Der Voort PHJ, et al. Serum and intraperitoneal levels of amphotericin B and flucytosine during intravenous treatment of critically III patients with candida peritonitis. J AntimicrobChemother. 2007;59(5):952-6.

24. Diekema DJ, et al. Activities of available and investigational antifungal agents against Rhodotorula species. J ClinMicrobiol. 2005;43(1):476-8.

25. Duggal $S$, et al. Rhodotorulaungemia: two cases and a brief review. Med Mycol. 2011;49:879-82

26. Li PKT, et al. International Society of Peritoneal Dialysis Recommendations: 2016 Update on Prevention and Treatment (2016) Peritoneal Dialysis International. 2016;36(5):481-508.

\section{Publisher's Note}

Springer Nature remains neutral with regard to jurisdictional claims in published maps and institutional affiliations.

Ready to submit your research? Choose BMC and benefit from:

- fast, convenient online submission

- thorough peer review by experienced researchers in your field

- rapid publication on acceptance

- support for research data, including large and complex data types

- gold Open Access which fosters wider collaboration and increased citations

- maximum visibility for your research: over $100 \mathrm{M}$ website views per year

At BMC, research is always in progress.

Learn more biomedcentral.com/submissions 